\title{
Personalia
}

\section{Prof. Dr. med. Egon Bücheler zum 60. Geburtstag}

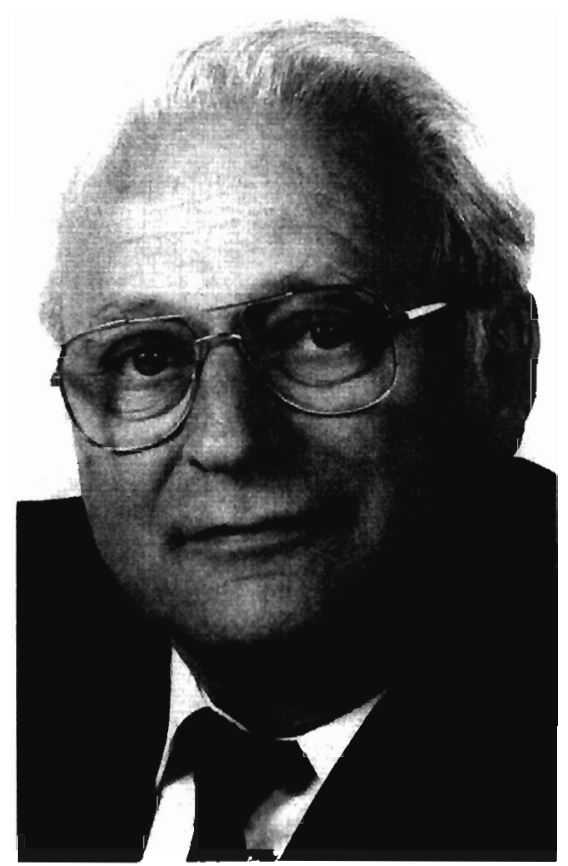

Am 12. Oktober 1994 wird Prof. Dr. med. Egon Bücheler, Direktor der Abteilung Röntgendiagnostik und Geschäftsführender Direktor der Radiologischen Klinik des Universitätskrankenhauses Hamburg-Eppendorl, 60 Jahre alt.

Egon Bücheler wurde z.u Köln geboren. Nach dem Studium der Medizin in Bonn, Hamburg und Zürich promovierte er 1960 in Bonn über ,Die Lungenembolie im Röntgenbild". Die Medizinalassistentenzeit absolvierte er an der Medizinischen und der Gynäkologischen Klinik der Universität und an der Chirurgischen Abteilung des St. Johannes-Hospitals in Bonn.

Seine Weitorbildung im Fach Radiologic begann 1962 an der Röntgenabteilung des Städtischen Krankenhauses Köln-Merheim bei Heigl. 1963 wurde er Assistenzarzt an der Röntgen- und Radiumklinik der Städtischen Krankenanstalten Aachen bei Thurn. Mit ihm, scinem geschätzten akademischen Lehrer wechselte er 1966 als wissenschaftlicher Assistent an die Radiologische Universitätsklinik in Bonn. Im Jahre 1968 erhielt or die Venia legendi für das Fach Radiologie. Seinc Ernennung zum Professor erlolgte 1971. Schließlich wurde er

lortschr. Röntgenstr. 161,4 (1994) $382-383$

(C) Georg Thieme Verlag Stuttgart . New York
1974 aur den Lehrstuhl für Ritdiologie der Universität Hamburg als Nachlolger von H. Prévôt berufen.

Am Anfang seiner wissenschaftlichen Lauf bahn war die konventionelle Röntgendiagnostik bei Erkrankungen der Nicren und des Retroperitoneums sein thematischer Schwerpunkt. In der Folgezeit widmete er sich insbesondere der Angiographie, wobei die Diagnostik von Tumoren des Oberbauches und die portale liypertension im Vordergrund seines Interesses standen. Früh entdeckte or die Möglichkeiten, therapeutische angiographische Konzepte zu entwickeln. und erarbeitete unter anderem Methodik und klinischen Linsatz der Fmbolisation von Niere und Milz. Fr war einer der Wegbereiter der modernen interventionellen Radiologie.

Frühzeitig erkannte er dic vieltältigen Möglichkeiten der sich neu entwickelnden digitalen Verfahren. So integrierte er konsequent die digitale Subtraktionsangiographie, digitale Radiographie, Computertomographie. Sonographie und Magnetresonanztomographie in die klinische Diagnostik.

Das wissenschaftliche Werk von E. Bücheler ist vielseitig und von hohem Niveau. Weit über 200 Publikationen in national und international anerkannten Zeitschriften tragen seine Handschrilt, zahlreiche Buchbeiträge sind von ihm in bedeutenden Lehrbüchern der deutschsprachigen Radiologie verfaßt. Sein mit Thurn herausgegebenes Buch .,Einführung in die Radiologische Diagnostik" gilt als Standardwerk. Er ist Herausgeber bzw. Mitherausgeber einer Reihe wichtiger Büchor, die das große Spektrum der Diagnostischen Radiologie umfassen. Seit langen Jahren gibt Bücheler die Zcitschrift "Aktuelle Radiologie" heraus, deren Gestaltung or maBgeblich geprägt hat.

Von 1985 bis 1991 war er Vorsitzender der Sektion Diagnostische Radiologie in der Deutschen Röntgengesellschaft. Den 73. Deutschen Röntgenkongreß in Wiesbaden 1992 leitete er erfolgreich als Kongreßpräsident. Seine langjährige Tätigkeit als Gutachter und Mitglied des Apparateausschusses der Deutschen Forschungsgemeinschaft ermöglichte ihm die aktive Weichenstellung der Forschung innerhalb der deutschen Radiologie.

Bücheler versteht sein Fachgebiet als klinische Disziplin. Fr sucht den Dialog mit den Vertretern der anderen klinischen Fächer zugunsten einer patientenorientierten medizinischen Krankenversorgung. Ausdruck dieses Verständnisses ist der von ihm inaugurierte jährlich stattfindende Kongreß in Hamburg .,Interdisziplínäre Gespräche in der Radiologie“. dessen Konzept inzwischen 
viele Kopien erfahren hat. Der große Zuspruch und die wachsenden Teilnehmerzahlen, dies gilt nicht nur für Radinlogen, bestätigten ihn in seiner Vorstcllung, daß dic Diagnostische und Interventionelle Radiologie ais klinisches Fach begrillen werden muß.

Sein unermüdlicher Einsatz fǜ dic radiologische Wissenschaft und die klinische Radiologie sind für seine Schüler und Mitarbeiter Vorbild. Ansporn und Motivation zugleich. Dabei überträgt er einerseits die an sich selbst gestellten hohen Anforderungen aul seine Mitarbeiter. für deren - auch persönliche - Belange er andererseits immer ein offenes ohr hat und für deren Zukunft er sich einsetzt. Seine Fntschlußfreudigkeit, gepaart mit pragmatischem Handeln, und seine Autorität machen ihn zum Vorbild für seine Schüler. Sein breitgefächertes Wissen auf allen Gebieten der diagnostischen Radiologie ließen ihn zum geschätzten akademischen Lehrer werden.
Wir, seine ehemaligen und jetzigen Mitarbeiter, Schüler und Freunde, gratulieren herzlichst und in Dankbarkeit.

W. Crone-Münzebrock/llamburg M. Heller/Kiel 